\title{
CONVERGENCE ACCELERATION FOR THE NUMERICAL SOLUTION OF SECOND-ORDER LINEAR RECURRENCE RELATIONS*
}

\author{
PAUL LEVRIE $\dagger \ddagger$ AND ADHEMAR BULTHEEL $\dagger$
}

\begin{abstract}
The concept of modification used for accelerating the convergence of ordinary continued fractions is adapted to the case of the Gautschi-Aggarwal-Burgmeier algorithm for the computation of nondominant solutions of nonhomogeneous second-order linear recurrence relations.
\end{abstract}

Key words. convergence acceleration, recurrence relation, continued fraction

AMS(MOS) subject classifications. 65Q05, 65B99, 40A15

1. Introduction. One of the first algorithms for the calculation of nondominant solutions of linear recurrence relations was developed by Gautschi [4]-[6]. The algorithm is based on the relationship between recurrence relations and continued fractions. The method is only applicable in the case of a second-order linear homogeneous recurrence relation. In 1978 Aggarwal and Burgmeier [1] generalized this algorithm to the case of nonhomogeneous recurrence relations (see also Van der Cruyssen [23]). As we will show, this algorithm is closely related to Olver's method of solving second-order recurrence relations [14], more specifically to Van der Cruyssen's reformulation of Olver's algorithm in terms of an LU-decomposition [22] (see also Cash [3], Lozier [13]).

In some cases the convergence of both of these algorithms is slow. The classical way to solve this problem is by using asymptotic information about the wanted solution of the recurrence relation (see, for instance, Gautschi [4], Scraton [19]—several authors have developed techniques to obtain the asymptotic behavior of all solutions of a linear recurrence relation; see Wimp [24]). The Gautschi algorithm (and its generalization to nonhomogeneous recurrence relations) lends itself very well to careful analysis when asymptotic values are used. It will be possible to prove convergence acceleration explicitly.

In $\S 2$ we give a derivation of the generalized Gautschi algorithm and discuss its relationship with Olver's algorithm [14], [22]. In $\S 3$ we define a modification of this algorithm and in $\S 4$ we show how this modification may be used to accelerate the convergence of the method. For homogeneous higher-order recurrence relations the same method has been used in [2], [10]-[12]. Finally, in $\S 5$ we give some examples.

2. Definitions and notation. Consider the second-order linear nonhomogeneous recurrence relation

$$
y_{n+2}+a_{1}(n) y_{n+1}+a_{2}(n) y_{n}=a_{3}(n), \quad n=0,1, \cdots
$$

with $a_{2}(n) \neq 0$. We assume that the homogeneous equation associated with (1):

$$
y_{n+2}+a_{1}(n) y_{n+1}+a_{2}(n) y_{n}=0, \quad n=0,1, \cdots
$$

has a minimal solution; this is a solution $f_{n}$ such that for every other solution $g_{n}$ of (2), linearly independent of $f_{n}$, we have

$$
\lim _{n \rightarrow \infty} \frac{f_{n}}{g_{n}}=0 .
$$

\footnotetext{
* Received by the editors October 21, 1987; accepted for publication (in revised form) December 9, 1988.

$\dagger$ Department of Computer Science, Katholieke Universiteit Leuven, Celestijnenlaan 200A, B-3030 Heverlee, Belgium.

\$ Katholieke Industriële Hogeschool Antwerpen, Salesianenlaan 1a, B2710 Hoboken, Belgium.
} 
(Let $f_{0} \neq 0$.) Furthermore, we assume that the recurrence relation (1) has a solution $h_{n}$ for which

$$
\lim _{n \rightarrow \infty} \frac{h_{n}}{g_{n}}=0
$$

with $g_{n}$ as defined above.

We denote by $A_{n}^{(1)}$ and $A_{n}^{(2)}$ the solutions of (2) with initial values:

$$
\begin{array}{ll}
A_{0}^{(1)}=1, & A_{1}^{(1)}=0, \\
A_{0}^{(2)}=0, & A_{1}^{(2)}=1
\end{array}
$$

and by $A_{n}$ the solution of (1) with

$$
A_{0}=0, \quad A_{1}=0 .
$$

A solution $p_{n}$ of (1) of the form

$$
p_{n}=C f_{n}+h_{n}, \quad n=0,1, \cdots
$$

for some constant $C \neq 0$ is uniquely defined by one initial condition. Forward recurrence with (1) is not a stable method to compute $p_{n}$. For the calculation of $p_{n}$ we construct a first-order recurrence relation (Aggarwal and Burgmeier [1], Van der Cruyssen [23]):

$$
y_{n+1}=b_{1}(n) y_{n}+b_{2}(n), \quad n=0,1, \cdots,
$$

which has a general solution of the form (5). To determine the coefficients in this recurrence relation we proceed as follows.

From (6) we get

$$
\begin{aligned}
y_{n+2} & =b_{1}(n+1) y_{n+1}+b_{2}(n+1) \\
& =b_{1}(n+1)\left(b_{1}(n) y_{n}+b_{2}(n)\right)+b_{2}(n+1) .
\end{aligned}
$$

Substituting the expressions (6) and (7) into (1) gives us

$$
\left(\left(a_{1}(n)+b_{1}(n+1)\right) b_{1}(n)+a_{2}(n)\right) y_{n}=a_{3}(n)-b_{2}(n+1)-\left(a_{1}(n)+b_{1}(n+1)\right) b_{2}(n) \text {. }
$$

Since every solution of (6) is a solution of (1) we must have

$$
\begin{aligned}
& \left(a_{1}(n)+b_{1}(n+1)\right) b_{1}(n)+a_{2}(n)=0, \\
& a_{3}(n)-b_{2}(n+1)-\left(a_{1}(n)+b_{1}(n+1)\right) b_{2}(n)=0 .
\end{aligned}
$$

We rewrite these expressions as follows:

$$
\begin{aligned}
& b_{1}(n)=-\frac{a_{2}(n)}{a_{1}(n)+b_{1}(n+1)}, \\
& b_{2}(n)=\frac{a_{3}(n)-b_{2}(n+1)}{a_{1}(n)+b_{1}(n+1)} .
\end{aligned}
$$

Note that equation (8a) generates an ordinary continued fraction: in the case of a homogeneous recurrence relation the given method reduces to the continued fraction method of Gautschi [4]-[6]. As in the method of Gautschi approximations to the coefficients $b_{1}(n), b_{2}(n)$ are obtained by calculating for an integer $N$ :

$$
\begin{aligned}
& b_{1, N}(N+1)=b_{2, N}(N+1)=0, \\
& b_{1, N}(n)=-\frac{a_{2}(n)}{a_{1}(n)+b_{1, N}(n+1)}, \\
& b_{2, N}(n)=\frac{a_{3}(n)-b_{2, N}(n+1)}{a_{1}(n)+b_{1, N}(n+1)}, \quad n=N, N-1, \cdots, 0 .
\end{aligned}
$$


If we want to calculate the solution $p_{n}$ of (1) starting with the initial value $p_{0}$, we get an approximation $p_{n}^{(N)}$ of $p_{n}$ using

$$
p_{0}^{(N)}=p_{0}, \quad p_{n+1}^{(N)}=b_{1, N}(n) p_{n}^{(N)}+b_{2, N}(n), \quad n=0,1, \cdots, N,
$$

in the sense that

$$
\lim _{N \rightarrow \infty} p_{n}^{(N)}=p_{n}
$$

for all values of $n$. The stability of this method has been proved in [10], [13].

Algorithm (9)-(10) is closely related to the algorithm first developed by Olver in [14] and reformulated in terms of an LU-decomposition by Van der Cruyssen [22]. To see this note that $p_{n}^{(N)}(n=0, \cdots, N)$ in $(10)$ is a solution of the following system of equations:

$$
\begin{aligned}
& z_{0}=p_{0}, \\
& a_{2}(n) z_{n}+a_{1}(n) z_{n+1}+z_{n+2}=a_{3}(n), \quad n=0, \cdots, N, \\
& z_{N+2}=0,
\end{aligned}
$$

or

$$
\left(\begin{array}{ccccccc}
1 & 0 & & & & \\
a_{2}(0) & a_{1}(0) & 1 & & & \\
& a_{2}(1) & a_{1}(1) & 1 & & \\
& & & \cdots & & & \\
& & & & a_{2}(N) & a_{1}(N) & 1 \\
& & & & & 0 & 1
\end{array}\right)\left(\begin{array}{c}
z_{0} \\
z_{1} \\
z_{2} \\
\vdots \\
z_{N+1} \\
z_{N+2}
\end{array}\right)=\left(\begin{array}{c}
p_{0} \\
a_{3}(0) \\
a_{3}(1) \\
\vdots \\
a_{3}(N) \\
0
\end{array}\right) .
$$

We decompose the matrix at the left-hand side in a product of an upper triangular and a lower triangular matrix:

$$
\left(\begin{array}{ccccccc}
1 & 0 & & & & & \\
& 1 & \alpha(0) & & & & \\
& 1 & \alpha(1) & & & \\
& & & \cdots & & \\
& & & & 1 & \alpha(N) \\
& & & & & & 1
\end{array}\right)\left(\begin{array}{cccccc}
1 & & & & & \\
a_{2}(0) & \beta(0) & & & \\
& a_{2}(1) & \beta(1) & & \\
& & \cdots & & \\
& & & a_{2}(N) & \beta(N) & \\
& & & & 0 & 1
\end{array}\right)
$$

with

$$
\begin{aligned}
& \alpha(N)=1, \quad \beta(N)=a_{1}(N), \\
& \alpha(i-1)=1 / \beta(i), \quad \beta(i-1)=a_{1}(i-1)-a_{2}(i) \alpha(i-1), \quad i=N, \cdots, 1 .
\end{aligned}
$$

Solving the system gives us

$$
z_{0}=p_{0}, \quad z_{i+1}=-\frac{a_{2}(i)}{\beta(i)} z_{i}+\frac{\gamma(i)}{\beta(i)}, \quad i=0, \cdots, N,
$$

with $\gamma(n)$ given by

$$
\gamma(N+1)=0, \quad \gamma(i)=a_{3}(i)-\alpha(i) \gamma(i+1), \quad i=N, \cdots, 0 .
$$


From these equations it is easy to deduce that

$$
b_{1, N}(n)=-\frac{a_{2}(n)}{\beta(n)}, \quad b_{2, N}(n)=\frac{\gamma(n)}{\beta(n)}
$$

for $n=0, \cdots, N$.

In the Olver-Van der Cruyssen method the system (12) is solved using an LUdecomposition (without pivoting). The method (9)-(10) is equivalent to using an LU-decomposition to solve the system

$$
\begin{aligned}
& \dot{z}_{0}=0, \\
& \dot{z}_{n}+a_{1}(N-n) \dot{z}_{n+1}+a_{2}(N-n) \dot{z}_{n+2}=a_{3}(N-n), \quad n=0, \cdots, N, \\
& \dot{z}_{N+2}=p_{0}
\end{aligned}
$$

(i.e., the system (12) with the order of the equations reversed) and then putting

$$
z_{i}=\dot{z}_{N+2-i}, \quad i=0, \cdots, N+2 .
$$

Remark. We note that algorithm (9)-(10) fails if one of the $\beta(i)$ in (13) is equal to zero. This only occurs if one of the following matrices:

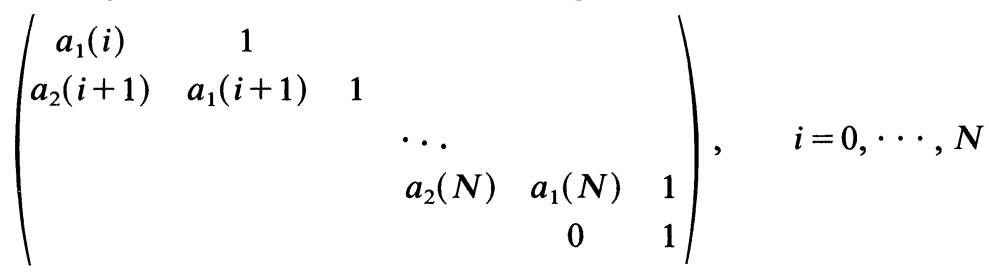

is singular (Horn and Johnson [7]). In most cases this problem can be solved by increasing $N$. For a detailed analysis, see Gautschi [4].

In the rest of this paper our main interest will lie in the calculation of $p_{1}$ given $p_{0}$. Once $p_{1}$ is known, the same method, i.e., (10), may be applied to calculate $p_{2}$ from $p_{1}$, and so on.

3. Modification and convergence of the algorithm. In algorithm (9) we have chosen the value zero as an approximation to $b_{1}(N+1)$ and $b_{2}(N+1)$. The choice is rather arbitrary and as we will see in the fourth paragraph modified approximants for $b_{1}(0)$ and $b_{2}(0)$ resulting from other choices than zero will often lead to acceleration of the convergence in (11). To introduce these modified approximants, we define transformations $s_{n}(u), t_{n}(u, v), S_{n}(u)$, and $T_{n}(u, v)$ by

$$
\begin{aligned}
& s_{n}(u)=-\frac{a_{2}(n)}{a_{1}(n)+u}, \quad t_{n}(u, v)=\frac{a_{3}(n)-v}{a_{1}(n)+u}, \quad n=0,1, \cdots, \\
& S_{0}(u)=s_{0}(u), \quad T_{0}(u, v)=t_{0}(u, v), \\
& S_{n}(u)=S_{n-1}\left(s_{n}(u)\right), \quad T_{n}(u, v)=T_{n-1}\left(s_{n}(u), t_{n}(u, v)\right), \quad n=1,2, \cdots .
\end{aligned}
$$

Then it is easy to prove that our previous approximants resulted from setting $b_{1, N}(N+1)=u=0$ and $b_{2, N}(N+1)=v=0$ :

$$
b_{1, N}(0)=S_{N}(0), \quad b_{2, N}(0)=T_{N}(0,0) .
$$

More generally, if

$$
\begin{aligned}
& b_{1, N}(N+1)=u, \quad b_{2, N}(N+1)=v, \\
& b_{1, N}(n)=-\frac{a_{2}(n)}{a_{1}(n)+b_{1, N}(n+1)},
\end{aligned}
$$




$$
b_{2, N}(n)=\frac{a_{3}(n)-b_{2, N}(n+1)}{a_{1}(n)+b_{1, N}(n+1)}, \quad n=N, N-1, \cdots, 0
$$

then

$$
b_{1, N}(0)=S_{N}(u), \quad b_{2, N}(0)=T_{N}(u, v) .
$$

Hence $S_{n}(u)$ and $T_{n}(u, v)$ are the approximations to $b_{1}(0)$ and $b_{2}(0)$ that we get if we replace $b_{1}(n+1)$ and $b_{2}(n+1)$ in $(8)$ by $u$ and $v$, respectively, and then use (8) to compute $b_{1}(0)$ and $b_{2}(0)$ backward. $S_{n}(u)$ and $T_{n}(u, v)$ are called modified approximants to $b_{1}(0)$ and $b_{2}(0)$.

We now have the following theorem.

THEOREM 1. For $k=0,1, \cdots$

$$
\begin{aligned}
& S_{k}(u)=-\frac{A_{k+2}^{(1)}-A_{k+1}^{(1)} u}{A_{k+2}^{(2)}-A_{k+1}^{(2)} u}, \\
& T_{k}(u, v)=-\frac{A_{k+2}-A_{k+1} u-v}{A_{k+2}^{(2)}-A_{k+1}^{(2)} u} .
\end{aligned}
$$

Proof. We use induction on $k$. For $k=0$ the right-hand side of equation (16b) is equal to

$$
-\frac{A_{2}-A_{1} u-v}{A_{2}^{(2)}-A_{1}^{(2)} u}=-\frac{a_{3}(0)-v}{-a_{1}(0)-u}
$$

and this expression is equal to $T_{0}(u, v)$. For $k>0$ let us assume that (16b) is true for $k=m-1$; then for $k=m$ we have

$$
\begin{aligned}
T_{m}(u, v) & =T_{m-1}\left(s_{m}(u), t_{m}(u, v)\right) \\
& =T_{m-1}\left(-\frac{a_{2}(m)}{a_{1}(m)+u}, \frac{a_{3}(m)-v}{a_{1}(m)+u}\right) \\
& =-\frac{A_{m+1}\left(a_{1}(m)+u\right)+A_{m} a_{2}(m)-a_{3}(m)+v}{A_{m+1}^{(2)}\left(a_{1}(m)+u\right)+A_{m}^{(2)} a_{2}(m)} \\
& =-\frac{a_{1}(m) A_{m+1}+a_{2}(m) A_{m}-a_{3}(m)+A_{m+1} u+v}{a_{1}(m) A_{m+1}^{(2)}+a_{2}(m) A_{m}^{(2)}+A_{m+1}^{(2)} u} \\
& =-\frac{-A_{m+2}+A_{m+1} u+v}{-A_{m+2}^{(2)}+A_{m+1}^{(2)} u}
\end{aligned}
$$

since $A_{n}$ and $A_{n}^{(2)}$ are solutions of (1) and (2), respectively.

The same method may be used to prove (16a).

As a consequence of this theorem we have

$$
S_{n}(0)=-\frac{A_{n+2}^{(1)}}{A_{n+2}^{(2)}}, \quad T_{n}(0,0)=-\frac{A_{n+2}}{A_{n+2}^{(2)}} .
$$

The method (9)-(10) is said to converge if the limits for $n \rightarrow \infty$ of the right-hand sides in (17) exist. If the conditions stated in the beginning of the previous paragraph ((3), $(4)$, and $\left.f_{0} \neq 0\right)$ are satisfied, then it is possible to prove convergence [10], [13]. Throughout this paper we assume that those conditions are satisfied.

4. Convergence acceleration. We want to calculate

$$
p_{1}=b_{1}(0) p_{0}+b_{2}(0) \text {. }
$$

A first method to do this is given by (9) and (10). In some cases, however, the convergence of this algorithm will be slow. For a good choice of $u$ and $v$ in (15) the convergence will be accelerated. 
Let us define

$$
U_{n}(u, v)=p_{0} S_{n}(u)+T_{n}(u, v)
$$

with $p_{0}$ the initial value for the solution $p_{n}$ of (1). We then have the following theorem.

THEOREM 2. For $k=0,1, \cdots$ and for arbitrary $u$ and $v$

$$
\begin{aligned}
& \frac{b_{1}(0)-S_{k}(u)}{b_{1}(0)-S_{k}(0)}=\left(1-\frac{A_{k+1}^{(2)}}{A_{k+2}^{(2)}} u\right)^{-1}\left(1-\frac{f_{k+1}}{f_{k+2}} u\right), \\
& \frac{b_{2}(0)-T_{k}(u, v)}{b_{2}(0)-T_{k}(0,0)}=\left(1-\frac{A_{k+1}^{(2)}}{A_{k+2}^{(2)}} u\right)^{-1}\left(1-\frac{\left(\beta_{1} f_{k+1}+h_{k+1}\right) u+v}{\beta_{1} f_{k+2}+h_{k+2}}\right), \\
& \frac{p_{1}-U_{k}(u, v)}{p_{1}-U_{k}(0,0)}=\left(1-\frac{A_{k+1}^{(2)}}{A_{k+2}^{(2)}} u\right)^{-1}\left(1-\frac{p_{k+1} u+v}{p_{k+2}}\right)
\end{aligned}
$$

with $\beta_{1}=-h_{0} / f_{0}$.

Proof. We will prove (18c). There exist numbers $\alpha_{1}, \alpha_{2}, \beta_{1}, \beta_{2}$ such that

$$
A_{n}=\alpha_{1} A_{n}^{(2)}+\beta_{1} f_{n}+h_{n}, \quad A_{n}^{(1)}=\alpha_{2} A_{n}^{(2)}+\beta_{2} f_{n} .
$$

Since

$$
\lim _{n \rightarrow \infty} \frac{A_{n}^{(1)}}{A_{n}^{(2)}}=-b_{1}(0), \quad \lim _{n \rightarrow \infty} \frac{A_{n}}{A_{n}^{(2)}}=-b_{2}(0),
$$

we get for the numerator of the left-hand side of equation $(18 \mathrm{c})$ :

$$
\begin{aligned}
p_{1}-U_{k}(u, v) & =p_{0}\left(b_{1}(0)-S_{k}(u)\right)+b_{2}(0)-T_{k}(u, v) \\
& =\lim _{n \rightarrow \infty}\left(p_{0}\left(-\frac{A_{n}^{(1)}}{A_{n}^{(2)}}-S_{k}(u)\right)-\frac{A_{n}}{A_{n}^{(2)}}-T_{k}(u, v)\right) .
\end{aligned}
$$

Using Theorem 1 and (19), for the first term on the right we get

$$
\begin{aligned}
\lim _{n \rightarrow \infty}( & \left.-\frac{A_{n}^{(1)}}{A_{n}^{(2)}}-S_{k}(u)\right) \\
& =\lim _{n \rightarrow \infty}\left(\frac{A_{k+2}^{(1)}-A_{k+1}^{(1)} u}{A_{k+2}^{(2)}-A_{k+1}^{(2)} u}-\frac{A_{n}^{(1)}}{A_{n}^{(2)}}\right) \\
& =\lim _{n \rightarrow \infty}\left(\frac{\alpha_{2} A_{k+2}^{(2)}+\beta_{2} f_{k+2}-\left(\alpha_{2} A_{k+1}^{(2)}+\beta_{2} f_{k+1}\right) u}{A_{k+2}^{(2)}-A_{k+1}^{(2)} u}-\frac{\alpha_{2} A_{n}^{(2)}+\beta_{2} f_{n}}{A_{n}^{(2)}}\right) \\
& =\lim _{n \rightarrow \infty}\left(\frac{\beta_{2}\left(f_{k+2} A_{n}^{(2)}-f_{n} A_{k+2}^{(2)}-\left(f_{k+1} A_{n}^{(2)}-f_{n} A_{k+1}^{(2)}\right) u\right)}{A_{n}^{(2)}\left(A_{k+2}^{(2)}-A_{k+1}^{(2)} u\right)}\right) \\
& =\lim _{n \rightarrow \infty}\left(\beta_{2}\left(A_{k+2}^{(2)}-A_{k+1}^{(2)} u\right)^{-1}\left(f_{k+2}-\frac{f_{n}}{A_{n}^{(2)}} A_{k+2}^{(2)}-\left(f_{k+1}-\frac{f_{n}}{A_{n}^{(2)}} A_{k+1}^{(2)}\right) u\right)\right) \\
& =\left(\beta_{2}\left(A_{k+2}^{(2)}-A_{k+1}^{(2)} u\right)^{-1}\left(f_{k+2}-f_{k+1} u\right)\right)
\end{aligned}
$$

since

$$
\lim _{n \rightarrow \infty} \frac{f_{n}}{A_{n}^{(2)}}=0 .
$$

Using the same method we get for the second term

$$
\lim _{n \rightarrow \infty}\left(-\frac{A_{n}}{A_{n}^{(2)}}-T_{k}(u, v)\right)=\left(A_{k+2}^{(2)}-A_{k+1}^{(2)} u\right)^{-1}\left(\beta_{1} f_{k+2}+h_{k+2}-\left(\beta_{1} f_{k+1}+h_{k+1}\right) u-v\right)
$$


and hence

$p_{1}-U_{k}(u, v)=\left(A_{k+2}^{(2)}-A_{k+1}^{(2)} u\right)^{-1}\left(\left(\beta_{2} p_{0}+\beta_{1}\right) f_{k+2}+h_{k+2}-\left(\left(\beta_{2} p_{0}+\beta_{1}\right) f_{k+1}+h_{k+1}\right) u-v\right)$.

From this it follows that

$$
p_{1}-U_{k}(0,0)=\left(A_{k+2}^{(2)}\right)^{-1}\left(\left(\beta_{2} p_{0}+\beta_{1}\right) f_{k+2}+h_{k+2}\right) .
$$

From the initial conditions for $A_{n}^{(1)}, A_{n}^{(2)}$, and $A_{n}$ it is easy to deduce that

$$
\beta_{1}=-\frac{h_{0}}{f_{0}}, \quad \beta_{2}=\frac{1}{f_{0}},
$$

and hence we have

$$
\left(\beta_{2} p_{0}+\beta_{1}\right) f_{n}+h_{n}=\frac{p_{0}-h_{0}}{f_{0}} f_{n}+h_{n}=p_{n} .
$$

The last equality is true for all $n$ since it is true for $n=0$. We now have

$$
\frac{p_{1}-U_{k}(u, v)}{p_{1}-U_{k}(0,0)}=\left(\frac{A_{k+2}^{(2)}-A_{k+1}^{(2)} u}{A_{k+2}^{(2)}}\right)^{-1} \frac{p_{k+2}-p_{k+1} u-v}{p_{k+2}},
$$

and this proves $(18 \mathrm{c})$. The same argument may be used to prove (18a) and (18b).

It is easy to see from (15) that

$$
S_{n}\left(b_{1}(n+1)\right)=b_{1}(0), \quad T_{n}\left(b_{1}(n+1), b_{2}(n+1)\right)=b_{2}(0)
$$

for all $n$. This is an extreme convergence acceleration. In general we do not know the exact values of $b_{1}(n+1)$ and $b_{2}(n+1)$ but in many cases it is possible to find approximations $u_{n}$ and $v_{n}$ to $b_{1}(n+1)$ and $b_{2}(n+1)$. Using these often leads to better approximations $S_{n}\left(u_{n}\right)$ and $T_{n}\left(u_{n}, v_{n}\right)$ to $b_{1}(0)$ and $b_{2}(0)$ than $S_{n}(0)$ and $T_{n}(0,0)$.

In the following two cases we have convergence acceleration:

$$
\begin{aligned}
& \text { (20a) Case (i) } \lim _{k \rightarrow \infty} \frac{b_{1}(0)-S_{k}\left(u_{k}\right)}{b_{1}(0)-S_{k}(0)}=0 \text { and } \lim _{k \rightarrow \infty} \frac{b_{2}(0)-T_{k}\left(u_{k}, v_{k}\right)}{b_{2}(0)-T_{k}(0,0)}=0 ; \\
& \text { (20b) Case (ii) } \lim _{k \rightarrow \infty} \frac{p_{1}-U_{k}\left(u_{k}, v_{k}\right)}{p_{1}-U_{k}(0,0)}=0 .
\end{aligned}
$$

By using Theorem 2 it is easy to select values for $u_{k}$ and $v_{k}$ that will lead to convergence acceleration. We will always assume that the first factor in equations $(18 \mathrm{a})-(18 \mathrm{c})$ satisfies

$$
\left|1-\frac{A_{k+1}^{(2)}}{A_{k+2}^{(2)}} u_{k}\right|>M>0
$$

for some $M$ and from some $k$ on.

The classical method to accelerate the convergence of the algorithm (see, e.g., Piessens [18]) is solving the system (12) with $z_{N+2}=0$ replaced by $z_{N+2}=p_{N+2}^{\text {(asym) }}$ where $p_{n} \sim p_{n}^{(\text {asym) }}(n \rightarrow \infty)$ (this means that $\left.\lim _{n \rightarrow \infty} p_{n}^{(\text {asym) }} / p_{n}=1\right)$. This is equivalent with the choice

$$
u_{N}=0, \quad v_{N}=p_{N+2}^{(\text {asym })}
$$

as can be seen from (9)-(10).

It is not always easy to obtain expressions for the asymptotic behavior of the solutions of a recurrence relation although methods are available (Wimp [24]). The following theorem due to Perron [15] gives us some information about the asymptotic behavior of the solutions of a homogeneous linear recurrence relation. 
THEOREM (Perron). Let $r$ be a natural number and let

$$
y_{n+r}+e_{1}(n) y_{n+r-1}+e_{2}(n) y_{n+r-2}+\cdots+e_{r}(n) y_{n}=0
$$

$(n=0,1, \cdots)$ be a linear rth-order homogeneous recurrence relation, with

$$
e_{r}(n) \neq 0 \quad \text { and } \quad \lim _{n \rightarrow \infty} e_{i}(n)=e_{i} \quad(i=1, \cdots, r) .
$$

If the auxiliary equation

$$
\rho^{r}+e_{1} \rho^{r-1}+e_{2} \rho^{r-2}+\cdots+e_{r}=0
$$

has roots $\rho_{1}, \cdots, \rho_{r}$ all with distinct moduli, then the given recurrence relation has nontrivial solutions $f_{n}^{(j)}(j=1, \cdots, r)$ such that

$$
\lim _{n \rightarrow \infty} \frac{f_{n+1}^{(j)}}{f_{n}^{(j)}}=\rho_{j} .
$$

An extension of this theorem to homogeneous recurrence relations of a more general nature has been proved by Perron [16]-[17] and Kreuser [9].

In our case, to get the asymptotic behavior of the solution $h_{n}$ of the nonhomogeneous equation (1) we construct the third-order homogeneous recurrence relation that has the three linearly independent solutions $f_{n}, g_{n}$, and $h_{n}$. This can be done if $a_{3}(n) \neq 0$ for all $n$ : from (1) we have

$$
\frac{y_{n+2}+a_{1}(n) y_{n+1}+a_{2}(n) y_{n}}{a_{3}(n)}=1 \quad \text { and } \quad \frac{y_{n+3}+a_{1}(n+1) y_{n+2}+a_{2}(n+1) y_{n+1}}{a_{3}(n+1)}=1 .
$$

Since the right-hand sides of these equations are equal, the left-hand sides must also be equal, and after rearranging we find

$$
\begin{aligned}
y_{n+3} & +\left(a_{1}(n+1)-\frac{a_{3}(n+1)}{a_{3}(n)}\right) y_{n+2} \\
& +\left(a_{2}(n+1)-\frac{a_{3}(n+1)}{a_{3}(n)} a_{1}(n)\right) y_{n+1}-\frac{a_{3}(n+1)}{a_{3}(n)} a_{2}(n) y_{n}=0 .
\end{aligned}
$$

Then we use Perron's theorem or the methods described in [24] to determine the asymptotic behavior of $h_{n}$.

We look at some special cases.

Case (1). $\lim _{n \rightarrow \infty} h_{h} / f_{n}=\gamma$ for some number $\gamma$. From (5) we have $p_{n} \sim(C+\gamma) f_{n}$, $(n \rightarrow \infty)$. If we take $u_{k} \sim f_{k+2} / f_{k+1}(k \rightarrow \infty)$ and $v_{k}=0$, then we get convergence acceleration (Case (i)). To see this rewrite the second factor in (18b):

$$
1-\frac{\left(\beta_{1} f_{k+1}+h_{k+1}\right) u_{k}+v_{k}}{\beta_{1} f_{k+2}+h_{k+2}}=1-\frac{\beta_{1}+h_{k+1} / f_{k+1}}{\beta_{1}+h_{k+2} / f_{k+2}} \frac{f_{k+1}}{f_{k+2}} u_{k}
$$

and this proves (20a) under the condition that $\beta_{1}+\gamma \neq 0$.

Case (2). $\lim _{n \rightarrow \infty} f_{n} / h_{n}=0$. In this case we choose $u_{k} \sim h_{k+2} / h_{k+1}(k \rightarrow \infty)$ and $v_{k}=0$. Since

$$
\lim _{k \rightarrow \infty} \frac{p_{k+2}}{p_{k+1}} / \frac{h_{k+2}}{h_{k+1}}=1,
$$

we get $(20 b)$.

Case (3). $a_{3}(n)=0$, for all $n\left(\Rightarrow h_{n} \equiv 0\right)$. If the given recurrence relation is homogeneous, then the problem is reduced to the calculation of the value of an ordinary continued fraction. Techniques for convergence acceleration of continued fractions may be found in [8] and [21] and the references given there. 
Some remarks. (1) We note that when using the method described above to determine the asymptotic behavior of $h_{n}\left(\right.$ or $\left.p_{n}\right)$, we only get this up to a constant factor (since the third-order recurrence relation used is homogeneous). To determine this factor is not always easy. In the method (21) this factor is needed. However, if we use the modification described in Cases (1) and (2) it is easy to see that the factor cancels out.

(2) If the solution $p_{n}$ is rapidly converging to zero, using the asymptotic expansion in (21) will cause problems when $\left|p_{N+2}^{(\text {asym) }}\right|$ is smaller than $\varepsilon_{\min }$. This will not occur if we use the modifications from Cases (1) and $(2) .\left(\varepsilon_{\min }\right.$ is the smallest positive real number representable on the computer.)

\section{Two examples.}

Example 1. Consider the recurrence relation (1) with coefficients given by

$$
\begin{aligned}
& a_{1}(n)=-2-\frac{1}{(10 n+11)^{2}} \\
& a_{2}(n)=0.99+\frac{1}{(n+1)^{3}} \\
& a_{3}(n)=4 \exp (-1)
\end{aligned}
$$

The asymptotic behavior of the solutions $f_{n}$ and $g_{n}$ of the associated homogeneous equation is given by

$$
\lim _{n \rightarrow \infty} \frac{f_{n+1}}{f_{n}}=0.9, \quad \lim _{n \rightarrow \infty} \frac{g_{n+1}}{g_{n}}=1.1
$$

and for the solution $h_{n}$ we have

$$
\lim _{n \rightarrow \infty} \frac{h_{n+1}}{h_{n}}=1 .
$$

This is an example of Case (2). We have calculated an approximation to $p_{1}$ (with $p_{0}=1$ ) using four different choices for $u_{k}$ and $v_{k}$ (see Tables 1(a)-1(c)-calculations with 16 significant figures):

(1) $u_{k}=0, v_{k}=0$, for all $k$ (second column).

(2) $u_{k}=1, v_{k}=0$, for all $k$ (third column).

(3) $u_{k}=0, v_{k}=-400 / e$, for all $k$ (fourth column).

(4) $u_{k}=0.9, v_{k}=-40 / e$, for all $k$ (fifth column).

The first choice gives the original method (9)-(10), the second choice is the one described in the previous paragraph (Case (2)). If we want to use the classical method (21), we need more information about the solution $p_{n}$. Now, using a paper by Späth [20] it is possible to prove that for this example $p_{n}$ satisfies

$$
\lim _{n \rightarrow \infty} p_{n}=-\frac{400}{e} \text {. }
$$

The third choice is given by (21) with $p_{n}^{\text {(asym) }}=-400 / e$.

As a consequence of Perron's theorem we have $\lim _{n \rightarrow \infty} b_{1}(n)=0.9$. Since $p_{n}$ is a solution of equation (6) we have

$$
\lim _{n \rightarrow \infty} b_{2}(n)=\lim _{n \rightarrow \infty} p_{n+1}-\lim _{n \rightarrow \infty} b_{1}(n) \lim _{n \rightarrow \infty} p_{n}=-\frac{400}{e}-0.9\left(-\frac{400}{e}\right)=-\frac{40}{e} .
$$


TABLE 1(a)

$b_{1}(0)=2.109233363$.

\begin{tabular}{rccc}
\hline \multicolumn{1}{c}{$n$} & $S_{n}(0)$ & $S_{n}(1)$ & $S_{n}(0.9)$ \\
\hline 50 & 2.109208819 & 2.109263364 & 2.109233361 \\
60 & 2.109230063 & 2.109237397 & 2.109233363 \\
100 & 2.109233362 & 2.109233365 & 2.109233363 \\
110 & 2.109233363 & 2.109233364 & 2.109233363 \\
120 & 2.109233363 & 2.109233363 & 2.109233363 \\
\hline
\end{tabular}

TABLE 1(b)

$b_{2}(0)=-18.74679823$.

\begin{tabular}{rcccc}
\hline \multicolumn{1}{c}{$n$} & $T_{n}(0,0)$ & $T_{n}(1,0)$ & $T_{n}(0,-400 / e)$ & $T_{n}(0.9,-40 / e)$ \\
\hline 90 & -18.74081420 & -18.74679804 & -18.74679838 & -18.74679822 \\
100 & -18.74449109 & -18.74679821 & -18.74679825 & -18.74679823 \\
110 & -18.74590873 & -18.74679823 & -18.74679824 & -18.74679823 \\
120 & -18.74645529 & -18.74679823 & -18.74679823 & -18.74679823 \\
250 & -18.74679822 & -18.74679823 & -18.74679823 & -18.74679823 \\
260 & -18.74679823 & -18.74679823 & -18.74679823 & -18.74679823 \\
\hline
\end{tabular}

TABLE $1(\mathrm{c})$

$p_{1}=-16.63756486$.

\begin{tabular}{ccccc}
\hline$n$ & $U_{n}(0,0)$ & $U_{n}(1,0)$ & $U_{n}(0,-400 / e)$ & $U_{n}(0.9,-40 / e)$ \\
\hline 100 & -16.63525773 & -16.63756485 & -16.63756487 & -16.63756487 \\
110 & -16.63667537 & -16.63756486 & -16.63756487 & -16.63756487 \\
120 & -16.63722193 & -16.63756486 & -16.63756487 & -16.63756487 \\
130 & -16.63743265 & -16.63756486 & -16.63756486 & -16.63756486 \\
230 & -16.63756485 & -16.63756486 & -16.63756486 & -16.63756486 \\
240 & -16.63756486 & -16.63756486 & -16.63756486 & -16.63756486 \\
\hline
\end{tabular}

By using this in Theorem 2 it is easy to prove that the fourth choice for $u_{k}$ and $v_{k}$ leads to convergence acceleration.

Note that the convergence of $S_{n}(1)$ is not as fast as that of $S_{n}(0)$ and $S_{n}(0.9)$. However, for the computation of $p_{1}$ the sequence $U_{n}(1,0)$ converges faster than all the other methods, although less information was used about $p_{n}$ than in the third and fourth choices for $u_{k}$ and $v_{k}$.

Example 2. Consider the recurrence relation (1) with coefficients given by

$$
\begin{aligned}
& a_{1}(n)=-4 \frac{n+2}{2 n+3}-\frac{1}{50(n+1)^{3}}, \\
& a_{2}(n)=\frac{2 n+5}{2 n+3}, \\
& a_{3}(n)=0.5^{n+1} \ln (n+2) .
\end{aligned}
$$

Then the asymptotic behavior of the solutions $f_{n}$ and $g_{n}$ is given by

$$
\lim _{n \rightarrow \infty} f_{n}=c_{1} \in \mathbb{R}, \quad \lim _{n \rightarrow \infty} \frac{g_{n}}{n^{2}}=c_{2} \in \mathbb{R}
$$


and for the solution $h_{n}$ using Perron's theorem, we find

$$
\lim _{n \rightarrow \infty} \frac{h_{n+1}}{h_{n}}=0.5
$$

Numerical experiments show that

$$
\lim _{n \rightarrow \infty} h_{n}=-0.42957 \cdots .
$$

This is an example of Case (1). In Tables 2(a)-2(c) we have calculated an approximation to $p_{1}\left(p_{0}=1\right)$ using three different choices for $u_{k}$ and $v_{k}$ :

(1) $u_{k}=0, v_{k}=0$, for all $k$ (second column-see (9)-(10)).

(2) $u_{k}=1, v_{k}=0$, for all $k$ (third column-see case (2)).

(3) $u_{k}=0, v_{k}=-0.42957$, for all $k$ (fourth column-see (21)).

We note that if the second term in the definition of $a_{1}(n)$ is omitted, then the resulting recurrence relation has the minimal solution $f_{n}=1$ for all $n$.

TABLE 2(a)

$b_{1}(0)=0.9866939003$.

\begin{tabular}{rcc}
\hline$n$ & $S_{n}(0)$ & $S_{n}(1)$ \\
\hline 100 & 0.9864215590 & 0.9866939094 \\
200 & 0.9866238022 & 0.9866939014 \\
300 & 0.9866624382 & 0.9866939006 \\
400 & 0.9866761154 & 0.9866939004 \\
500 & 0.9866824842 & 0.9866939003 \\
1000 & 0.9866910292 & 0.9866939003 \\
5000 & 0.9866937849 & 0.9866939003 \\
\hline
\end{tabular}

TABLE 2(b)

$b_{2}(0)=-0.4288098297$.

\begin{tabular}{rccc}
\hline$n$ & $T_{n}(0,0)$ & $T_{n}(1,0)$ & $T_{n}(0,-0.42957)$ \\
\hline 100 & -0.4284182552 & -0.4288098428 & -0.4285374796 \\
200 & -0.4287090421 & -0.4288098314 & -0.4287397303 \\
300 & -0.4287645933 & -0.4288098302 & -0.4287783672 \\
400 & -0.4287842585 & -0.4288098299 & -0.4287920447 \\
500 & -0.4287934156 & -0.4288098298 & -0.4287984135 \\
1000 & -0.4288057017 & -0.4288098297 & -0.4288069587 \\
5000 & -0.4288096638 & -0.4288098297 & -0.4288097143 \\
\hline
\end{tabular}

TABLE 2(c)

$p_{1}=0.5578840705$.

\begin{tabular}{rccc}
\hline$n$ & $U_{n}(0,0)$ & $U_{n}(1,0)$ & $U_{n}(0,-0.42957)$ \\
\hline 100 & 0.5580033038 & 0.5578840665 & 0.5578840794 \\
200 & 0.5579147601 & 0.5578840700 & 0.5578840718 \\
300 & 0.5578978449 & 0.5578840704 & 0.5578840710 \\
400 & 0.5578918569 & 0.5578840705 & 0.5578840707 \\
500 & 0.5578890686 & 0.5578840705 & 0.5578840706 \\
1000 & 0.5578853275 & 0.5578840705 & 0.5578840706 \\
5000 & 0.5578841211 & 0.5578840705 & 0.5578840705 \\
\hline
\end{tabular}




\section{REFERENCES}

[1] V. B. Aggarwal and J. W. Burgmeier, An algorithmic decomposition system for second order recurrence relations, BIT, 18 (1978), pp. 241-258.

[2] M. G. DE BRUIN AND L. JACOBSEN, Modification of Generalised Continued Fractions I, Lecture Notes in Math. 1237, Springer-Verlag, Berlin, New York, 1987, pp. 161-176.

[3] J. R. CASH, An extension of Olver's method for the numerical solution of linear recurrence relations, Math. Comp., 32 (1978), pp. 497-510.

[4] W. GAuTSCHI, Computational aspects of three-term recurrence relations, SIAM Rev., 9 (1967), pp. $24-82$.

[5] — Zur numerik rekurrenter relationen, Computing, 9 (1972), pp. 107-126.

[6] - Computational methods in special functions-a survey, in Theory and Application of Special Functions, R. A. Askey, ed., Academic Press, New York, San Francisco, 1975, pp. 1-98.

[7] R. A. Horn And C. R. Johnson, Matrix Analysis, Cambridge University Press, Cambridge, 1985.

[8] L. JACOBSEN, Modified approximants for continued fractions, construction and applications, Skript. K. Nor. Vidensk. Selsk., 3 (1983), pp. 1-46.

[9] P. KREUSER, Uber das Verhalten der Integrale homogener linearer Differenzengleichungen im Unendlichen, Ph.D. thesis, University of Tubingen, Leipzig, East Germany, 1914.

[10] P. LEVRIE, Het numeriek oplossen van lineaire recursiebetrekkingen: een veralgemening van de kettingbreukmethode van Gautschi, Ph.D. thesis, Katholieke Universiteit Leuven, Belgium, 1987.

[11] P. Levrie AND R. Piessens, Convergence acceleration for Miller's algorithm, in Nonlinear Numerical Methods and Rational Approximation, A. Cuyt, ed., Reidel, Dordrecht, the Netherlands, 1987, pp. 349-370.

[12] P. LEVRIE AND L. JACOBSEN, Convergence acceleration for generalized continued fractions, Trans. Amer. Math. Soc., 305 (1988), pp. 263-276.

[13] D. W. LozIER, Numerical solution of linear difference equations, Report NBSIR 80-1976, National Bureau of Standards, U.S. Dept. of Commerce, Washington, D.C., 1980.

[14] F. W. J. OLVER, Numerical solution of second order linear difference equations, J. Res. Nat. Bur. Standards, 71 (1967), pp. 111-129.

[15] O. Perron, Uber einen Satz des Herrn Poincaré, J. Reine Angew. Math., 136 (1909), pp. 17-37.

[16] _ Uber Summengleichungen und Poincarésche Differenzengleichungen, Math. Ann., 84 (1921), pp. $1-15$.

[17] - Uber lineare Differenzengleichungen und eine Anwendung auf lineare Differentialgleichungen mit Polynomkoeffizienten, Math. Z., 72 (1959), pp. 16-24.

[18] R. Piessens, Modified Clenshaw-Curtis integration and applications to numerical computation of integral transforms, in Numerical Integration. Recent Developments, Software and Applications, P. Keast and G. Fairweather, eds., Reidel, Dordrecht, the Netherlands, 1987, pp. 35-51.

[19] R. E. SCRAton, A modification of Miller's recurrence algorithm, BIT, 12 (1972), pp. 242-251.

[20] H. SPÄTH, Uber das asymptotische Verhalten der Lösungen nichthomogener linearer Differenzengleichungen, Acta Math., 51 (1927), pp. 133-199.

[21] W. J. THRON AND H. WAADELAND, Accelerating convergence of limit-periodic continued fractions $K\left(a_{n} / 1\right)$, Numer. Math., 34 (1980), pp. 155-170.

[22] P. VAN DER CRUySSEn, A reformulation of Olver's algorithm for the numerical solution of second-order linear difference equations, Numer. Math., 32 (1979), pp. 159-166.

[23] - Nonhomogeneous recursions and generalised continued fractions, BIT, 22 (1982), pp. 533-537.

[24] J. Wimp, Computation with Recurrence Relations, Pitman, Boston, London, 1984. 